\title{
Mathematical Model of Instability Phenomenon with Magnetic Fluid through Homogeneous Porous Media
}

\author{
N. B. Vyas ${ }^{a}$, M. N. Mehta ${ }^{\mathrm{b}}$, and T. R. Singh ${ }^{\mathrm{c}}$, \\ ${ }^{a}$ Department of Mathematics, CKPCET, Surat- 395007, India \\ ${ }^{\mathrm{b}, \mathrm{c}}$ Department of Mathematics, S.V. National Institute of Technology, Surat-395007, India
}

\begin{abstract}
In this paper the mathematical model of instability phenomenon occurs during secondary oil recovery process is formulated under the external magnetic effect which is due to injection of magnetic fluid into injection wells. Mathematical formulation of the present model leads tonon-linear partial differential equation of parabolic type which has been solved in form of power series with appropriate initial and boundary conditions for any time $T>0$. The solution represents saturation of injected phase for different time $T>0$. The graph shows that saturation of injected phase increases for distanceX increases for different time T. It is given by using MATLAB coding.
\end{abstract}

Keywords: Instability phenomenon, magnetic fluid, Power series solution

2010 Mathematics Subject Classification: 35Q35, 76S99

\section{Introduction}

The phenomenon of instability occurs during secondary oil recovery process while injecting fluid into injection well. This area of oil recovery is very vast and it is difficult to study exactly. So to study this type of complicated phenomenon the mathematical modelling is considered which is more effective for analysis purpose. To develop mathematical model of instability phenomenon some basic steps are considered. The mathematical model gives an idea or estimates the real phenomena. The mathematical modelling essentially consists of basic steps viz. translating present investigated problem into mathematical problem by using identification of variables, simplifying assumptions and accepting governing laws then solving this mathematical problem and interpreting the solution.

In this paper the phenomenon of instability that arises in displacement process involving two immiscible phases through homogeneous porous media with a layer of magnetic fluid injected into the displacing phase is discussed analytically under certain conditions. The basic assumptions underlying the investigation are, the displacement of native phase by another of lesser viscosity provides a well-developed finger flow, the displacing phase containing a layer of magnetic fluid is regarded as single phase Brailovsky [3], the magnetization $M$ is assumed directly proportional to the magnetic field intensity $H$ and the microscopic behaviour of fingers is governed by statistical treatment Scheidegger and Johnson [8].The objective for present investigation is to determine the saturation of injecting fluid occupied by fingers for any distance $\mathrm{x}$ and for any time $T>0$.

When one native phase $N$ is filled in pores of the porous medium and another phase $I$ is injected, which contains the layer of magnetic fluid then it increases the injecting phase velocity by $\left(\mu_{0} M+\frac{16 \pi \mu_{0} r^{3} \chi M}{9(l+2)^{3}}\right)\left(\frac{\partial H}{\partial x}\right)$, Verma and Rajput [14] where $\mu_{0}$ denotes the permeability of magnetic field

and it is not mixing with residential fluid $N$ which is non-conductive in ordinary condition, then instead of regular displacement of whole front atcommon interface protuberances take place which shoot through the porous medium at relatively very high speed. Thus instabilities occur in flow system depending on viscosity difference and wettability of these two fluids. Simply it can be defined when a fluid flowing through a porous medium is displaced by another fluid of lesser viscosity then fingers arise at common interface in the direction of resident fluid. This phenomenon is called instability or fingering phenomenon. Figure 1 represents the diagram of instabilities in cylindrical piece of porous media. It is assumed that variation in saturation of injected phase at common interface $x=0$ is very small $\varepsilon ; 0<\varepsilon<1$. Many researchers have discussed this phenomenon with a different point of view. Verma and Rajput [14] discussed the phenomenon of instabilities that arises in the displacement process involving two immiscible phases through dipping porous media with ferrofluid under certain conditions. Verma and Mishra [12]discussed Similarity solution for instabilities in double-phase flow through porous media. The displacement problems of this type in the porous medium have gained much current importance and many authors have analysed these problems; for example, Scheidegger [9], 
Verma [13], Rosenweig [7], Mehta [5] has discussed the instability phenomenon with different viewpoint. Lange et al. [4], Brailovsky et al. [3], Zhan and Yortos [16], Wang and Feyen [15] formulated nonlinear evolution equation for two phase displacement front and studied numerically.

Mathematical formulation of the investigated phenomena is more complicated. So it is assumed that, in addition to above assumptions, Darcy's law is valid for present flow phenomenon. In statistical treatment of fingers, only the behaviour of two phases involved is taken into consideration. The saturation of injected phase $S_{i}$ is defined as average cross-sectional area occupied by injected phase at distance $x$ i.e. $S_{i}(x, t)$. The mathematical formulation has been solved by using infinite Maclaurin's series. The co-efficient of infinite series consist of the magnetic field effect in this phenomenon for different time $T$.

\section{Statement of the problem}

Here we consider that there is a uniform injection of phase $I$, involving a layer of suitable magnetic fluid, into oil saturated homogeneous cylindrical piece of porous medium. The injecting phase with magnetic fluid is injected from one end of cylindrical piece of porous medium saturated with native phase $N$ of grater viscosity such that the injected phase cuts through oil formation at common interface $x=0$. Due to the layer of magnetic fluid the velocity of injected phase increases and gives rise to protuberance called instability (fingers). This furnishes well developed fingers, which is mentioned in Figure 1.

The area of secondary oil recovery is very vast so we consider the small part of this large area in form of cylindrical piece of oil saturated porous medium. For mathematical formulation of the model, the vertical cross section of this cylindrical porous medium is taken which is in form of rectangle and instability phenomenon in this rectangle area is given in figure 2. The main objective of the present paper is to find average cross sectional area occupied by the fingers of injected phase.

Since the entire native phase at the boundary $x=0$ is displaced through small distance as result of phase injection, it is assumed that the saturation of native phase exists at common boundary $x=0$. The saturation of injected phase at this boundary is very small $S_{i 0}$ and initial saturation is considered as $S_{i c}$.

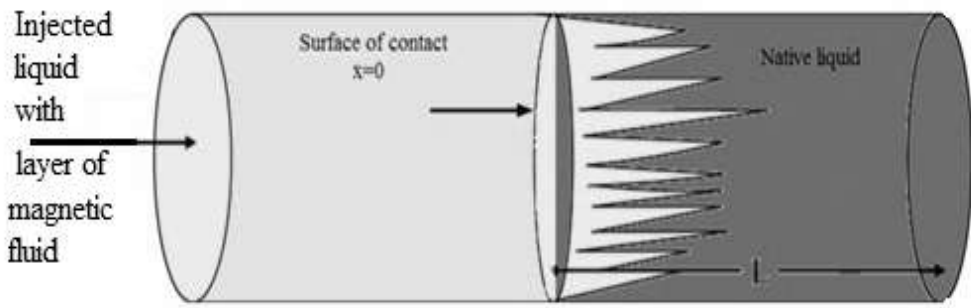

Figure 1: Formation of fingers in cylindrical piece of porous media with Layer of magnetic fluid

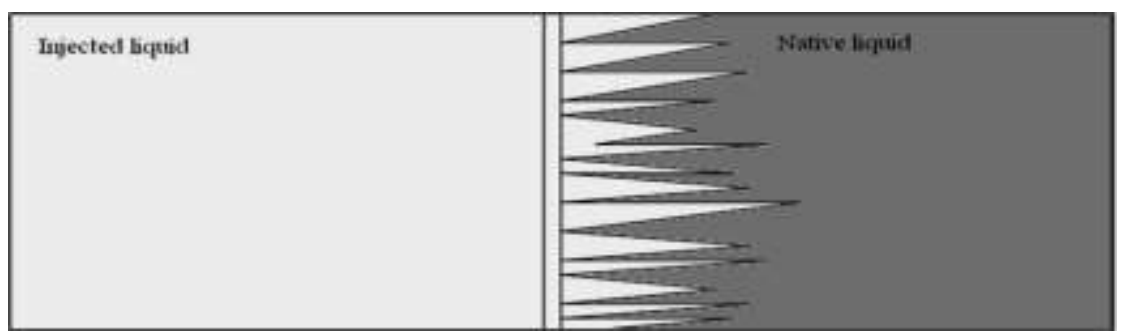

Figure 2: Instability phenomenon in vertical cross-sectional area

\section{Mathematical formulation}

Due to the increase of velocity in injected phase containing magnetic fluid, the native phase (oil) will be displaced through inter connected capillary. It is assumed that the flow is one-dimensional and it is governed by Darcy's law, the seepage velocity of injected phase and displaced native phase are expressed as, Bear [2].

$v_{i}=-\left(\frac{k_{i}}{\delta_{i}}\right) K\left(\frac{\partial p_{i}}{\partial x}\right)+\left(\mu_{0} M+\frac{16 \pi \mu_{0} r^{3} \chi M}{9(l+2)^{3}}\right)\left(\frac{\partial H}{\partial x}\right)$ 
$v_{n}=-\left(\frac{k_{n}}{\delta_{n}}\right) K\left(\frac{\partial p_{n}}{\partial x}\right)$

Where $\mathrm{K}$ is the permeability of the homogeneous medium, $k_{i}$ and $k_{n}$ are relative permeabilities of injected and native phases, which are functions $S_{i}$ and $S_{n}, p_{i}$ and $p_{n}$ are pressure, $\delta_{i}$ and $\delta_{n}$ are the constant kinematic viscosity of injected and native phases respectively.

It may be mentioned that the term $\left[\left(\mu_{0} M+\frac{16 \pi \mu_{0} r^{3} \chi M}{9(l+2)^{3}}\right)\left(\frac{\partial H}{\partial x}\right)\right]$ on right hand side of equation (1) represents the additional velocity exerted due to the presence of a layer of magnetic fluid in the displacing phase $I$.

The equation of continuity of two phases, when phase densities are regarded as constant, is given as, Bear [2],

$$
\begin{aligned}
& P\left(\frac{\partial S_{i}}{\partial t}\right)+\frac{\partial v_{i}}{\partial x}=0 \\
& P\left(\frac{\partial S_{n}}{\partial t}\right)+\frac{\partial v_{n}}{\partial x}=0
\end{aligned}
$$

Where $\mathrm{P}$ is the porosity of the medium.

From the definition of phase saturation,

$$
S_{i}+S_{n}=1
$$

The capillary pressure $p_{c}$ defined as the pressure discontinuity of the flowing phases across their common interface, is a function of the phase saturation. It may be written as,

$p_{c}=p_{n}-p_{i}$

$p_{c}=-\beta S_{i}$, Mehta [5].

Where $\beta$ is the constant capillary pressure coefficient and the negative sign indicates that the direction of injected phase is opposite to capillary pressure, Scheidegger [10]

For definiteness of the mathematical analysis the standard relation between phase saturation and relative permeability, given by Scheidegger and Johnson [8] is used here. It is given by,

$k_{i}=S_{i}$

$k_{n}=S_{n}=1-S_{i}$

The equation of motion for saturation is obtained by substituting the values of $v_{i}$ and $v_{n}$ from equations (1) and (2)

$$
\begin{aligned}
& p\left(\frac{\partial S_{i}}{\partial t}\right)+\frac{\partial}{\partial x}\left[-\left(\frac{k_{i}}{\delta_{i}}\right) K\left(\frac{\partial p_{i}}{\partial x}\right)+\left(\mu_{0} M+\frac{16 \pi \mu_{0} r^{3} \chi M}{9(l+2)^{3}}\right)\left(\frac{\partial H}{\partial x}\right)\right]=0 \\
& p\left(\frac{\partial S_{n}}{\partial t}\right)+\frac{\partial}{\partial x}\left[-K\left(\frac{k_{n}}{\delta_{n}}\right) \frac{\partial p_{n}}{\partial x}\right]=0
\end{aligned}
$$

On substituting the values of $\frac{\partial p_{i}}{\partial x}$ from (6) in (10), we get

$$
p\left(\frac{\partial S_{i}}{\partial t}\right)+\frac{\partial}{\partial x}\left[-\left(\frac{k_{i}}{\delta_{i}}\right) K\left(\frac{\partial p_{n}}{\partial x}-\frac{\partial p_{c}}{\partial x}\right)+\left(\mu_{0} M \frac{\partial H}{\partial x}+\frac{16 \pi \mu_{0} r^{3} \chi M}{9(l+2)^{3}} \frac{\partial H}{\partial x}\right)\right]=0
$$

Combining equations (11) and (12) by using equation (5) we get,

$$
\frac{\partial}{\partial x}\left[K\left(\frac{k_{i}}{\delta_{i}}+\frac{k_{n}}{\delta_{n}}\right) \frac{\partial p_{n}}{\partial x}-K\left(\frac{k_{i}}{\delta i}\right) \frac{\partial p_{c}}{\partial x}-\left(\mu_{0} M+\frac{16 \pi \mu_{o} r^{3} M \chi}{9(l+2)^{3}}\right)\left(\frac{\partial H}{\partial x}\right)\right]=0
$$

Integrating the above equation we get the following, 
$\left[K\left(\frac{k_{i}}{\delta_{i}}+\frac{k_{n}}{\delta_{n}}\right) \frac{\partial p_{n}}{\partial x}-K\left(\frac{k_{i}}{\delta i}\right) \frac{\partial p_{c}}{\partial x}-\mu_{0} M \frac{\partial H}{\partial x}-\frac{16 \pi \mu_{o} r^{3} M \chi}{9(l+2)^{3}} \frac{\partial H}{\partial x}\right]=V$

Here $V$ is the integrating constant which can be evaluated later.

On simplifying the above equation, the following is obtained,

$$
\frac{\partial p_{n}}{\partial x}=\frac{1}{K\left(\frac{k_{i}}{\delta_{i}}+\frac{k_{n}}{\delta_{n}}\right)}\left[V+K\left(\frac{k_{i}}{\delta_{i}}\right)\left(\frac{\partial p_{c}}{\partial x}\right)+\mu_{0} M \frac{\partial H}{\partial x}+\frac{16 \pi \mu_{o} r^{3} M \chi}{9(l+2)^{3}} \frac{\partial H}{\partial x}\right]
$$

From equation (12) the following is obtained,

$$
\begin{aligned}
& p\left(\frac{\partial S_{i}}{\partial t}\right)=\frac{\partial}{\partial x}[ {\left[\frac{\left(\frac{k_{i}}{\delta_{i}}\right)}{\left(\frac{k_{i}}{\delta_{i}}+\frac{k_{n}}{\delta_{n}}\right)}\left\{V+K\left(\frac{k_{i}}{\delta_{i}}\right)\left(\frac{\partial p_{c}}{\partial x}\right)+\mu_{0} M \frac{\partial H}{\partial x}+\frac{16 \pi \mu_{o} r^{3} M \chi}{9(l+2)^{3}} \frac{\partial H}{\partial x}\right\}\right.} \\
&\left.-K\left(\frac{k_{i}}{\delta_{i}}\right)\left(\frac{\partial p_{c}}{\partial x}\right)-\mu_{0} M \frac{\partial H}{\partial x}-\frac{16 \pi \mu_{o} r^{3} M \chi}{9(l+2)^{3}} \frac{\partial H}{\partial x}\right]
\end{aligned}
$$

The native pressure $\left(p_{n}\right)$ can be expressed as Oroveanu[6]

$$
p_{n}=\bar{p}+\frac{1}{2} p_{c}
$$

Where $\bar{p}$ is the constant mean pressure.

It may be mentioned here that the concept of mean pressure is justified in the statistical treatment of instability Verma [13].

On substituting the value of $\frac{\partial p_{n}}{\partial x}$ from equation (17) in equation (14) we get the following,

$V=\left[\frac{K}{2}\left(-\frac{k_{i}}{\delta_{i}}+\frac{k_{n}}{\delta_{n}}\right) \frac{\partial p_{c}}{\partial x}-\mu_{0} M \frac{\partial H}{\partial x}-\frac{16 \pi \mu_{o} r^{3} M \chi}{9(l+2)^{3}} \frac{\partial H}{\partial x}\right]$

On substituting the value of $V$ in equation (16), the following equation is obtained,

$$
p\left(\frac{\partial S_{i}}{\partial t}\right)+\frac{\partial}{\partial x}\left[\frac{K}{2}\left(\frac{k_{i}}{\delta_{i}}\right) \frac{\partial p_{c}}{\partial x}+\mu_{0} M \frac{\partial H}{\partial x}+\frac{16 \pi \mu_{o} r^{3} M \chi}{9(l+2)^{3}} \frac{\partial H}{\partial x}\right]=0
$$

For definiteness considering capillary pressure $p_{c}$ as a function of phase saturation and substituting the value of $p_{c}$ and $k_{i}$ from equation (7) and (8) in the above equation, the following equation is obtained,

$$
p\left(\frac{\partial S_{i}}{\partial t}\right)-\frac{\partial}{\partial x}\left[\frac{K \beta}{2 \delta_{i}}\left(S_{i} \frac{\partial S_{i}}{\partial x}\right)-\mu_{0} M \frac{\partial H}{\partial x}-\frac{16 \pi \mu_{o} r^{3} M \chi}{9(l+2)^{3}} \frac{\partial H}{\partial x}\right]=0
$$

This equation represents the governing equation of Instability phenomenon through homogeneous porous medium with magnetic fluid.

Considering $M=\chi H$ Verma and Rajput [15], $\chi=$ susceptibility, equation (20) reduces to,

$$
p\left(\frac{\partial S_{i}}{\partial t}\right)-\frac{\partial}{\partial x}\left[\frac{K \beta}{2 \delta_{i}}\left(S_{i} \frac{\partial S_{i}}{\partial x}\right)-\alpha\left(H \frac{\partial H}{\partial x}\right)\right]=0 ; \alpha=\left(\mu_{0} \chi+\frac{16 \pi \mu_{0} r^{3} \chi^{2}}{9(l+2)^{3}}\right)
$$

Considering the magnetic field intensity $H$ in $x$-direction only, Verma[13] and in such a way that it satisfies $H=\frac{\lambda}{x^{n}}$, Vermaand Rajput [14]where $\lambda$ is a constant and $n$ is an integer.

For the sack of definiteness and particular interest choose $n=-1$, Shah \&Verma [11]. 
Using the value of $H$ for $n=-1$ equation (21) is reduced to,

$p\left(\frac{\partial S_{i}}{\partial t}\right)-\frac{\partial}{\partial x}\left[\frac{K \beta}{2 \delta_{i}}\left(S_{i} \frac{\partial S_{i}}{\partial x}\right)-\alpha \lambda^{2} x\right]=0$

The appropriate set of boundary and initial conditions are given by Oroveanu [6],

$S_{i}(0, t)=S_{i 0}$ for $x=0, t>0$

$S_{i}(x, 0)=S_{i c}$ for $x>0, t=0$

$S_{i}(L, t)=S_{i 1}$ for $x=L, t>0$

\section{Solution of the Problem}

For more simplification, using the dimensionless variables given by,

$X=\frac{x}{L} \& T=\left(\frac{\beta k}{2 \delta_{i} L^{2} P}\right) t$

Equation (22) can be rewritten in the dimensionless form by considering above variables,

$\left(\frac{\partial S_{i}}{\partial T}\right)=\left[S_{i} \frac{\partial^{2} S_{i}}{\partial X^{2}}+\left(\frac{\partial S_{i}}{\partial X}\right)^{2}-A\right]$

Where, $A=\frac{2 \alpha \delta_{i} L^{2} \lambda^{2}}{\beta k}=\frac{2 \delta_{i} L^{2} \lambda^{2}}{\beta k}\left(\mu_{0} \chi+\frac{16 \pi \mu_{0} r^{3} \chi^{2}}{9(l+2)^{3}}\right)$

And set of initial and boundary conditions will be,

$$
\begin{aligned}
& S_{i}(0, T)=S_{i 0} ; X=0, T>0 \\
& S_{i}(X, 0)=S_{i c} ; X>0, T=0 \\
& S_{i}(L, T)=S_{i 1} ; X=L, T>0
\end{aligned}
$$

Choosing the similarity transform,

$$
S_{i}(X, T)=T f(\eta) ; \eta=\frac{X}{T}
$$

Equation (23) is reduced to,

$$
(-\eta) f^{\prime}(\eta)+f(\eta)=f(\eta) f^{\prime \prime}(\eta)+\left\{f^{\prime}(\eta)\right\}^{2}-A
$$

The corresponding boundary and initial conditions become,

$$
f(0)=\frac{S_{i 0}}{T} ; X=0, T>0
$$

The resulting problem with given condition at $T=0$ poses difficulties, because condition (25) is not easy to satisfy. So it can be transformed into the variational condition given by

$$
f^{\prime}(0)=\omega \neq 0 ; \text { (Small) } X>0, T=0
$$

Taking nth derivative of equation (28) and solving for $f^{(n+2)}(\eta)$ and evaluating at $\eta=0$ gives,

$$
\begin{aligned}
& f^{(n+2)}(0)=-\frac{1}{f(0)}\left[n f^{n}(0)\left\{1+f^{\prime \prime}(0)\right\}+(n+1) f^{\prime}(0) f^{n+1}(0)+\right. \\
& \left.\sum_{k=2}^{n}\left(\begin{array}{l}
n \\
k
\end{array}\right)\left\{f^{k+1}(0) f^{n-k+1}(0)+f^{k}(0) f^{n-k+2}(0)\right\}\right]
\end{aligned}
$$

For the solution, it is necessary to determine the derivatives $f^{(n)}(0)$ for all $n=1,2,3, \ldots \ldots$. The derivatives $f^{(1)}(0)$ can be determined by means of formula (30) and $f^{(2)}(0)$ from equation (28). Further all other derivatives can be determined from formula (31) for all $n \geq 1$.

Thus the desired value of $f(\eta)$ can be computed by Maclaurin's series, 


$$
f(\eta)=\sum_{k=0}^{\infty} f^{(k)}(0) \frac{\eta^{k}}{k !} ; f(\eta)=\frac{S_{i}(X, T)}{T} \& \eta=\frac{X}{T}
$$

This is an approximate solution of the mathematical model describing instability phenomenon of two immiscible fluids in homogeneous porous medium with magnetic fluid.The first two coefficients of this infinite series are given in equation (29) and in equation (30). Others are found by using these coefficients which are as follow,

$$
\begin{aligned}
& f(0)=\frac{S_{i 0}}{T} \\
& f^{(1)}(0)=\omega \\
& f^{(2)}(0)=\frac{S_{i 0}-\omega^{2} T+A T}{S_{i 0}} \\
& f^{(3)}(0)=\frac{-1}{\left(S_{i 0}\right)^{2}}\left[4 \omega T\left(S_{i 0}\right)-3 \omega^{3} T^{2}+3 T^{2} A \omega\right] \\
& f^{(4)}(0)=\frac{1}{\left(S_{i 0}\right)^{3}}\left[24 \omega^{2}\left(S_{i 0}\right) T^{2}-15 \omega^{4} T^{3}+18 A \omega^{2} T^{2}-8 A\left(S_{i 0}\right) T^{2}-5\left(S_{i 0}\right)^{2} T-3 A^{2} T^{3}\right]
\end{aligned}
$$

Using values of these coefficients, $f(\eta)$ and $\eta$ in equation (32), the series is reduced to,

$$
\begin{aligned}
& S_{i}(X, T)=S_{i 0}+X \omega+\frac{X^{2}}{2 T}\left[\frac{S_{i 0}-\omega^{2} T+A T}{S_{i 0}}\right]-\frac{X^{3}}{6 T^{2}}\left[\frac{4 \omega T\left(S_{i 0}\right)-3 \omega^{3} T^{2}+3 T^{2} A \omega}{\left(S_{i 0}\right)^{2}}\right]-\frac{X^{4}}{24 T^{3}} \\
& {\left[\frac{24 \omega^{2}\left(S_{i 0}\right) T^{2}-15 \omega^{4} T^{3}+18 A \omega^{2} T^{2}-8 A\left(S_{i 0}\right) T^{2}-5\left(S_{i 0}\right)^{2} T-3 A^{2} T^{3}}{\left(S_{i 0}\right)^{3}}\right]+\ldots}
\end{aligned}
$$

This equation represents the value of saturation of injected phase in the dimensionless variables. Using equation (33) and coefficients (A) to (E) the graphical representation is obtained.

\section{Graphical and Numerical Representation:}

The numerical values of saturation at time $T>0$ for different distances $0 \leq X \leq 1$ are given in the following table. It is obtained by considering equation (33) with coefficient (A) to (E) and also by taking the particular values of saturation at the distance $x=0$ i.e., $S_{i 0}$,variation in saturation $\omega$ and constants which is required for graphical representation of the present investigated phenomenon. The graphical representation is given by MATLAB coding. The following figure 3 shows the graph of Distance $X$ vs. Saturation $S_{i}(X, T)$ for different values of time $T>0$.

\begin{tabular}{|c|c|c|c|c|}
\hline Distance $X$ & \multicolumn{4}{|c|}{ Saturation $S_{i}(X, T)$} \\
\hline & $T=0.10$ & $T=0.11$ & $T=0.12$ & $T=0.13$ \\
\hline 0.0 & 0.1000 & 0.1000 & 0.1000 & 0.1000 \\
\hline 0.1 & 0.1107 & 0.1108 & 0.1110 & 0.1112 \\
\hline 0.2 & 0.1230 & 0.1234 & 0.1238 & 0.1247 \\
\hline 0.3 & 0.1367 & 0.1376 & 0.1385 & 0.1405 \\
\hline 0.4 & 0.1519 & 0.1535 & 0.1551 & 0.1587 \\
\hline 0.5 & 0.1685 & 0.1710 & 0.1736 & 0.1791 \\
\hline 0.6 & 0.1866 & 0.1902 & 0.1939 & 0.2019 \\
\hline 0.7 & 0.2062 & 0.2110 & 0.2161 & 0.2268 \\
\hline 0.8 & 0.2272 & 0.2335 & 0.2401 & 0.2540 \\
\hline 0.9 & 0.2497 & 0.2577 & 0.2659 & 0.2834 \\
\hline 1.0 & 0.2736 & 0.2834 & 0.2936 & 0.3150 \\
\hline
\end{tabular}


Table 1: Saturations of injected phase for different distance at different time $T>0$.

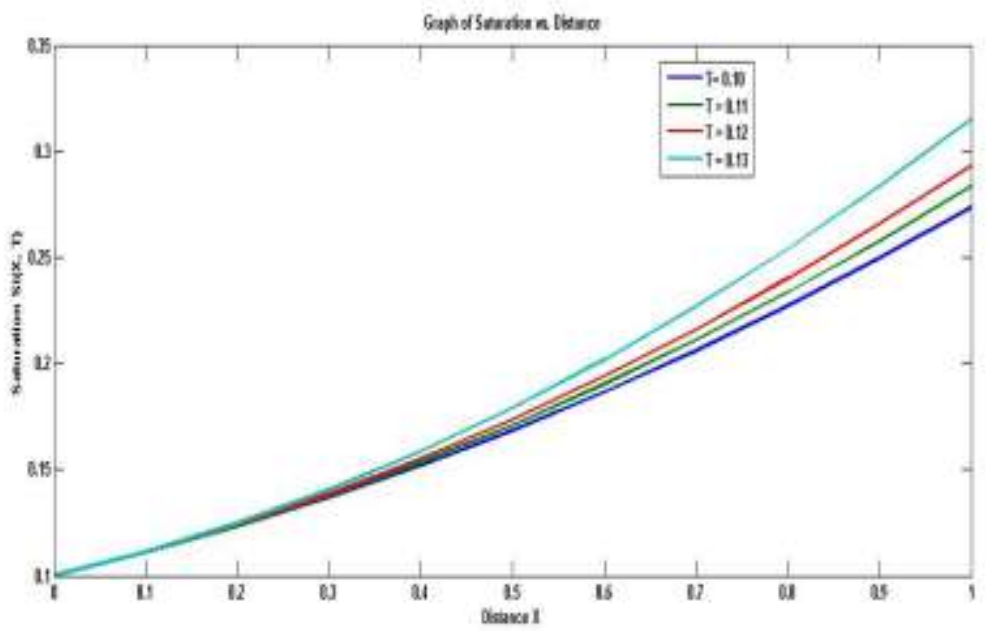

Figure 3: Plot of Distance vs. Saturation for $T=0.10,0.11,0.12,0.13$.

\section{Conclusions}

- Equation (32) with the coefficients (A) to (E) represents the solution of nonlinear problem of instability phenomenon under the injection of magnetic fluid which gives saturation of injected phase for different time $T$.

- Figure 3 gives the graph of equation (33) which is given for saturation $S_{i}(X, T)=T f(\eta)$ verses distance $X$ for different time $T=0.10,0.11,0.12,0.13$ respectively.

- When distance $X$ increases for $T>0$ the saturation $S_{i}(X, T)$ increases in each case and it is physically fact for instability phenomenon that fingers are increasing as distance increases.

- By considering, $u_{n}=f(\eta)=\sum_{k=0}^{\infty} f^{(k)}(0) \frac{\eta^{k}}{k !}$

$\Rightarrow u_{n+1}=\sum_{k=0}^{\infty} f^{(k+1)}(0) \frac{\eta^{k+1}}{(k+1) !}$

$\therefore \lim _{\eta \rightarrow \infty}\left|\frac{u_{n+1}}{u_{n}}\right|<1$

- So the equation (33) expressed in form of Maclaurin's series is convergent by ratio test, Bali and Iyengar [1].

\section{References}

[1] Bali and Iyengar. A Text book of engineering mathematics, $6^{\text {th }}$ Edition, Laxmi Publication Ltd, pp.592-593(2004).

[2] Bear J. Dynamics of fluids in porous media, American Elsevier, New York, (1972).

[3] Brailovsky I., Babchin A., Frankle M. and Sivashinsky G. Instability instability in water-oil displacement. Transport in porous media, No.63, 380-393(2006).

[4] Lang A., Schroter M., Schere M., Engel A. and Rehberg I. Instability instability in water-sand mixture. The European Physical Journal, No.4, 475-484,(1998).

[5] Mehta M. N. An asymptotic expansion in fluid flow through porous media; Ph.D. Thesis; S.G. University, Surat, India, (1978).

[6] Oroveanu T. ScurereaFluiidelorPrinMediPoeseaseNemogeme, EdituraAcadimiei R.P.R., Rumania, pp. 92 \&328, (1963).

[7] Rosenweig R. E., Berkovsky B. M., et al. Thermo mechanics of magnetic Fluid, (Hemisphere, Washington DC),pp. 195, (1978).

[8] Scheidegger A. E. and Johnson E. F. The statistical behavior of instabilities in displacement processes in porous media, Canadian Journal Physics, No. 39, 326-334(1961).

[9] Scheidegger A. E. Growth of instabilities on displacement fronts in porous media, Physics of Fluids, No. 3, 94(1960).

[10] Scheidegger A. E. The physics of flow through porous media, $3^{\text {rd }}$ ed., University of Toronto Press, Toronto, (1974).

[11] Shah R. C. \&Verma A. P. Fingro-Imbibition phenomenon through porous media with magnetic fluid, Indian Journal of Engg. \& Material Sciences, No.5, 411-415(1998).

[12] Verma A. P and Mishra S. K. Similarity solution for instabilities in double-phase flow through porous media. J.Appl.Phys No. 44, 1622 (1973).

[13] Verma A. P. Instabilities in two phase flow through porous media with magnetic fluids. In multiphase transport, Fundamentals, Reactor safety Applications, (Ed. T. N Veziroglu), Hamisphere publ. Co. Washington, No.5,1323-1335(1980). 
[14] Verma. A. P. and Rajput A. K. Instabilities in displacement process through porous media with magnetic fluid, J. Magnetism and Magnetic Materials North-Holland, Amsterdam, 65, 330-334(1987).

[15] Wang Z. and Feyen J. Prediction of instability in porous media. Water resources research, No.9, 2183-2190(1998).

[16] Zhan L. and Yortsos Y. C. The shape of a gravity finger in a rectangular channel in homogeneous porous media. Trans. Porous Media. No.49, 77-97(2002).

\section{Nomenclature}

$P=$ Porosity of the medium

$K=$ Permeability of the medium

$k_{n}=$ Relative permeability of native liquid

$k_{i}=$ Relative permeability of injected liquid

$\delta_{i}, \delta_{n}=$ Viscosity of injected and native liquid

$p_{i,} p_{n}=$ Pressures of injected and native liquid

$p_{c}=$ Capillary pressure

$S_{i}, S_{n}=$ Saturations of injected and native liquid

$v_{i}, v_{n}=$ Seepage velocity of injected and native liquid

$\beta=$ Capillary pressure coefficient

$\lambda=$ Constant

$H=$ Magnetic field intensity

$\mu_{0}=$ Magnetic field permeability

$M=$ Magnetization of the applied field

$\chi=$ Susceptibility

$r=$ Radius of magnetic fluid particles

$t=$ Time 